\title{
English Major Teaching and Evaluation of Test Results based on the Regression Model and SPSS
}

\author{
Xiaofang Qiu \\ Jincheng College of Sichuan University, Chengdu 611731, China
}

\begin{abstract}
In the paper, we conduct research on the English major teaching and evaluation of test results based on the regression model and SPSS. English classroom teaching evaluation is based on teaching objectives and teaching principles and the starting point, using the feasible evaluation technology in the English classroom teaching process and its effect to give value judgment. Learning a language is not an isolated process, college English teaching cannot ignore the import of the cultural background knowledge. College English teachers should help the students from English language phenomenon found in the culture. We propose the new evaluation model to enhance the performance of the traditional training methodology that will be meaningful.
\end{abstract}

Keywords: English Major; Teaching and Evaluation; Test Results; Regression Model and SPSS.

\section{Introduction}

College teachers as the main body of English teaching, teachers' knowledge level directly affects the quality of teaching in the process of college English teaching in China, high professional quality of young teachers or English major is less, this leads to a lot of English teachers' knowledge structure has certain problem, and with the continuous development of the times, the English teaching should also constantly advancing with the times to guide students, so that those who are interested in professional English students in the professional knowledge learning at the same time, increase the corresponding knowledge of English, and lay a good foundation for employment. According to the review, the core challenges for the modern English education can be summarized as the follows. (1) In a large class of middle school students English is hard to get the teacher's personality guidance, combined with the teaching material choice of the content, and backward, and boring and students themselves lack of self-confidence, it is difficult to find pleasure in learning English. (2) In the current college English teaching, most of the teachers and students have neglected the use and communication of this link led to the English teaching work, teaching, learning and the use. In English oral communication become insurmountable divide many college students. (3) Because of the college English course is scheduled as a basic course instead of professional course makes some English teachers in the teaching process conceptual deviation. They tend to put all of the teaching content to the final exam, just in order to make students well in the final exams, and some students have the same English class just to pass the exam to get the credits the negative thoughts.

English classroom teaching evaluation is based on teaching objectives and teaching principles and starting point, using the feasible evaluation technology in the English classroom teaching process and its effect to give value judgment. It undoubtedly has very important function for the English teaching evaluation significance and guiding value that hold the following functionalities.

- The selection guide function. For a unit of the English teaching, English classroom teaching evaluation for unit annual appraisal and evaluation of advanced model not only provides the basis, and more importantly that can be found in talents and training on the function of the selection and guide. The training and selection of the leader in the teaching unit, an important aspect of should see its ability to classroom teaching.

- Supervise control function and good habits of teaching methods and teaching to improve the quality of English teaching is very important. English teaching evaluation can play important role in urging. For those professional dedication targeted assessment of teachers is not strong can motivate them to set up the respect-work spirit to achieve good results. 
- Determination of feedback function. Due to assess to a series of quantitative and qualitative determination, such as analysis of the examination paper of calendar year performance curve, compare the students, and the evaluation test questions difficulty level and so on. The determination of the various feedbacks information can be obtained.

\section{Our Proposed Methodology}

\subsection{English Major Teaching Principles in Universities.}

In recent years, China's college English major teaching mode innovation becomes one of focuses of foreign language education. The characteristics of university teachers' English teaching knowledge could be then reflected from the following aspects.

- Language is not an isolated phenomenon of closed, but a spiritual, mental, cognitive, ethnic and economic, political and social factor intertwined open system, we need to have the aid of pedagogy, psychology, linguistics, cultural science, cognitive science and multi-disciplinary perspective to enrich the understanding of language teaching.

- Chinese teaching and English teaching is belonging to language teaching, but there is a difference. Linguistics theory to this effect is defined as "language transfer", regards it as a cross interlanguage effect is the result of the target language and that any other have the commonness and difference between the acquisitions of language.

- The most important function of language is communication, due to the language culture of feature, compared with the native language communication, cross interlanguage property took on a deeper partition English communication. In the process of the students use English for communication, pronunciation, vocabulary, grammar, language is only a part of barriers, such as a bigger obstacle comes from the cultural differences between east and west have different values, ways of the thinking, customs and other cultural factors will lead to the students' social pragmatic failures.

Traditional college English teaching is mainly solve the problem of students master English skills and knowledge of English, as to those related to the English language and cultural knowledge to students through the reading, watching or other methods to obtain, obviously ignored the import of cultural background knowledge. English language teaching must be closely combined with English culture teaching, and it is the only way to improve and enhance college English teaching in China. Learning a language is not an isolated process, college English teaching cannot ignore the import of the cultural background knowledge. College English teachers should help the students from English language phenomenon found in the culture, through mastering English culture to guide the study and practice, this not only embodies the teaching purpose and practical also can fully mobilize students' learning interest and the further development.

\subsection{Regression Model.}

Mathematic model is established based on the linear regression method, and the influence of the various periods of time in learning English, for example, using principal component analysis model systematically analyzes what time arrangement is more decisive role in learning English, and using linear regression analysis model got a prediction equation. Regardless of the independent variable is a category type variable or a continuous variable, the interaction are widespread in the contain multiple independent variables in the model. And empirical studies on various kinds of statistical teaching materials generally refer to interaction analysis of the factors of variance problem, but in discussing regression analysis are seldom involved in the interaction between continuous variables. Ignore the interaction between continuous variables will be the complexity of the relationship between fuzzy variables that not conducive to the construction of a theoretical model and validation.

Considering the students in the general professional class, between different professional learning environment, learning habits, teacher professor may exist such as aggregation, impact on the student individual professional English learning and assessment. So, first run the zero model tests between different professional gathering effects more levels in the logistic regression into the random effects 
processing hierarchy data related issues, in the group are different specialized students of professional English teaching effect evaluation of the random effects model as the formula one.

$$
\operatorname{logit}\left(p_{i j}\right)=\ln \left(\frac{p_{i j}}{1-p_{i j}}\right)=\gamma_{00}+\gamma_{0 i} W_{1 j}+\beta_{1} X_{1 i j}+\mu_{0 j}
$$

The visualized model is shown in the figure 1 .

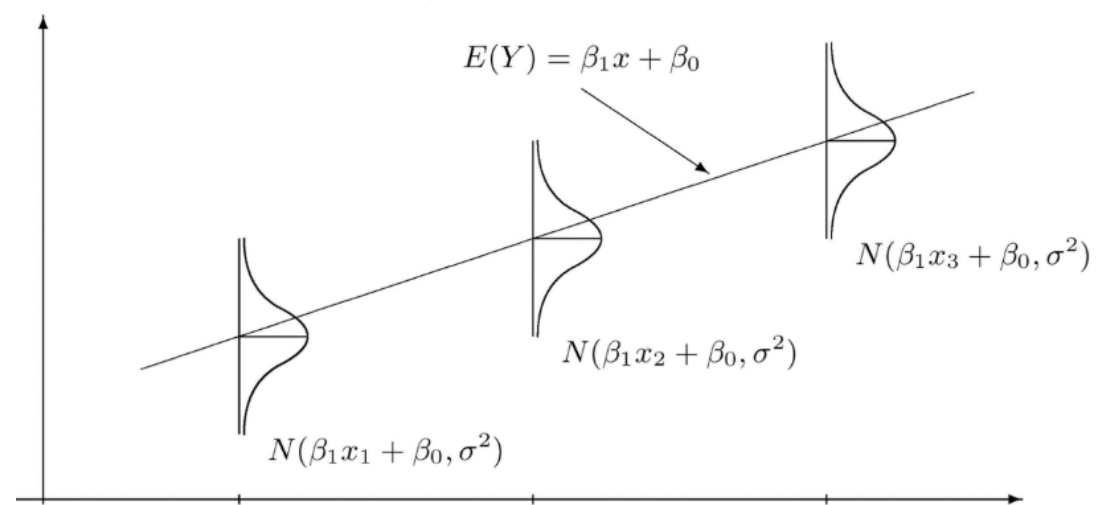

\subsection{English Education and SPSS.}

Fig. 1 the Visualized Demonstration of the Regression Model

For the analysis of English performance with the SPSS, we should follow the listed aspects. (1) Select the menu "Analyze the Data and the Reduction Factor", open the dialog box, Factor analysis to reflect the students' English learning motivation 20 involved in the analysis of the variable is selected, enter "Variables" box. (2) Click on the "Extraction" button and set the control parameters "Method" drop-down box to provide seven kinds of factor extraction Method, the use of system default principal component extraction method and specify the "Analyze" box "the Correlation matrix is adopted to improve the factor extraction. (3) In "factor analysis" dialog box, click the "Descriptive" button, the description statistics and the initial analysis results are set up, all the Settings, when confirmed click "OK" button, the output results are obtained shown as figure 2.

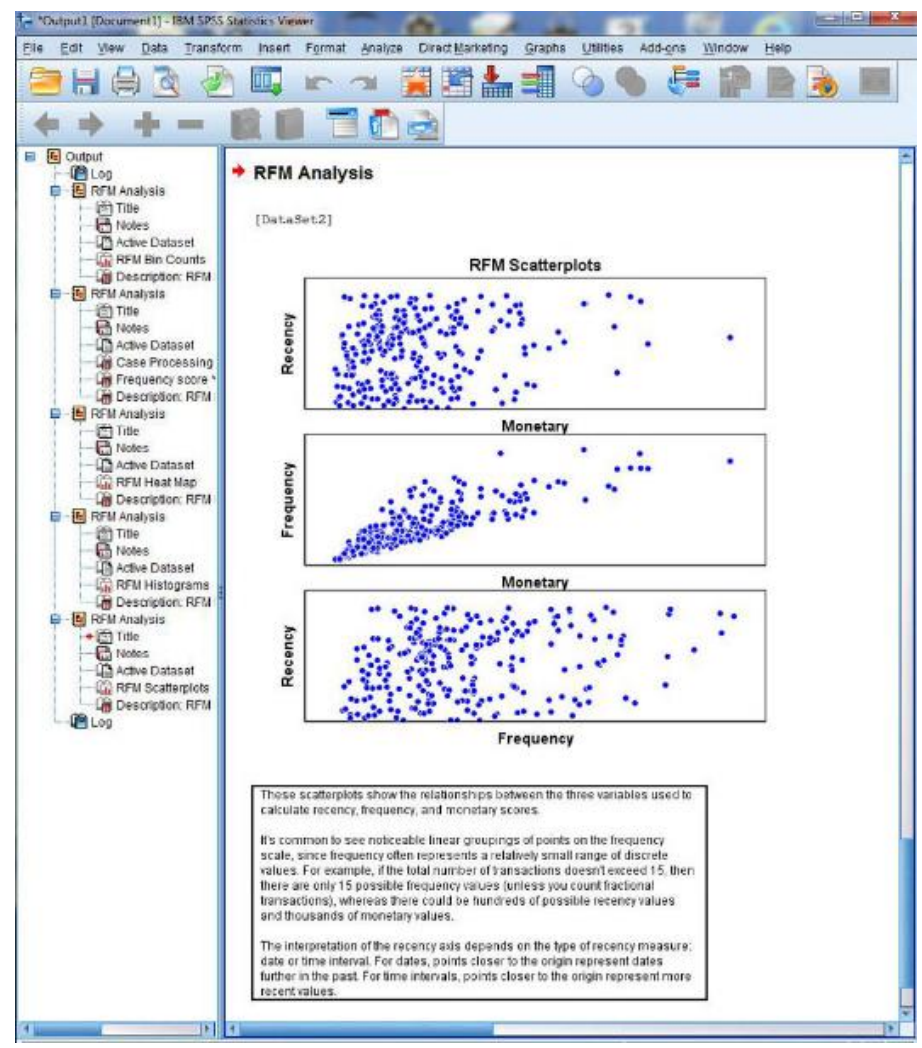

Fig. 2 The 2.3 English Education and SPSS Relationship Demonstration 


\subsection{English Teaching Test Evaluation.}

Language testing is with foreign language teaching. Language teaching cannot be separated from language testing, language tests, in turn, to produce a great "backwash effect of language teaching. Language teaching and language testing is inseparable. Psychological necessity and probability of the occurrence and development is the root cause of the development of the English education level to system. Students and teachers' psychological process of the occurrence and development are all in occurrence and development of the variable. Students' psychological variables are relatively simple, the occurrence and development levels is less, while the occurrence and development of teachers' psychology and general value occurred with specialized knowledge and skills of senior academic phenomenon in the occurrence and development. For the evaluation, we should consider following aspects. (1) Even adulthood, native speakers of vocabulary knowledge continues to increase, but its syntactic knowledge seldom development. (2) To understand a word means knowing the context of the possibility of the encounter with other word. (3) To understand a word means knowing the result of the function and situation changes the use of the limited extent. (4) To understand a word means knowing syntactic pattern associated with this word. (5) To understand a word means knowing the change form and the derivative forms. (6) To understand a word means knowing the relationship associated with other words in a language.

\section{Conclusion}

In the paper, we conduct research on the English major teaching and evaluation of test results based on the regression model and SPSS. English classroom teaching evaluation is based on teaching objectives and teaching principles and starting point, using the feasible evaluation technology in the English classroom teaching process and its effect to give value judgment. It undoubtedly has very important function for the English teaching evaluation significance and guiding value. We integrate the statistical model to conduct the reasonable evaluation that is meaningful.

\section{References}

[1] Yu, Dejian. "Triangular hesitant fuzzy set and its application to teaching quality evaluation." Journal of Information and Computational Science 10.7 (2013): 1925-1934.

[2] Huber, Elaine, and Marina Harvey. "Time to participate: Lessons from the literature for learning and teaching project evaluation in higher education." Studies in Educational Evaluation 39.4 (2013): 240-249.

[3] Liu, Zongxiang. "A Comprehensive Evaluation Method for Physical Education Teaching Based on Analytic Hierarchy Process." International Journal of Digital Content Technology and its Applications 7.2 (2013): 404-411.

[4] Bergsmann, Evelyn, et al. "Evaluation of competence-based teaching in higher education: From theory to practice." Evaluation and program planning 52 (2015): 1-9.

[5] Cawthorn, Thomas R., et al. "Development and evaluation of methodologies for teaching focused cardiac ultrasound skills to medical students." Journal of the American Society of Echocardiography 27.3 (2014): 302-309. 